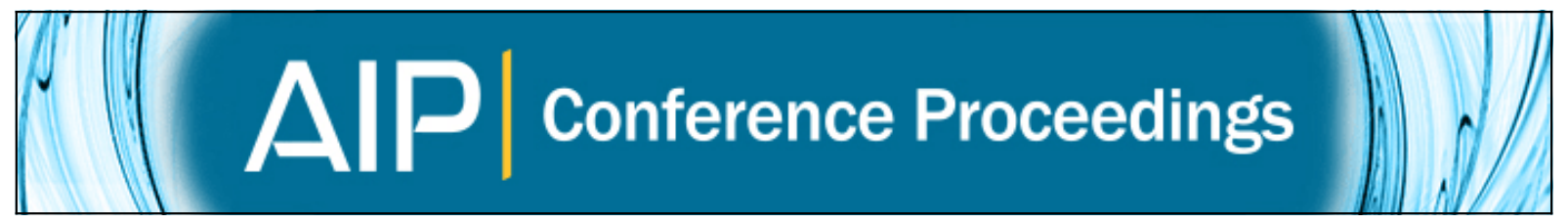

A new challenge for meteorological measurements: The "MeteoMet" project - Metrology for meteorology

A. Merlone, G. Lopardo, I. Antonsen, S. Bell, R. Benyon, N. Boese, D. del Campo, M. Dobre, J. Drnovšek, A. Elkatmis, E. Georgin, E. Grudniewicz, M. Heinonen, C. Holstein-Rathlou, J. Johansson, P. Klason, R. Knorova, C. Melvad, J. Merrison, K. Migała, M. de Podesta, H. Saathoff, D. Smorgon, F. Sparasci, R. Strnad, A. SzmyrkaGrzebyk, and E. Vuillermoz

Citation: AIP Conference Proceedings 1552, 1030 (2013); doi: 10.1063/1.4821419

View online: http://dx.doi.org/10.1063/1.4821419

View Table of Contents: http://scitation.aip.org/content/aip/proceeding/aipcp/1552?ver=pdfcov

Published by the AIP Publishing

Articles you may be interested in

Thermodynamic analysis and experimental study of the effect of atmospheric pressure on the ice point AIP Conf. Proc. 1552, 221 (2013); 10.1063/1.4819543

Changes in surface irradiance and meteorological parameters associated with the annular solar Eclipse of 15 January 2010

AIP Conf. Proc. 1531, 600 (2013); 10.1063/1.4804841

General approach to the formulation and solution of the multi-parameter inverse problems of atmospheric remote sensing

AIP Conf. Proc. 1531, 240 (2013); 10.1063/1.4804751

Characterization of particle hygroscopicity by Raman lidar: Selected case studies from the convective and orographically-induced precipitation study

AIP Conf. Proc. 1531, 204 (2013); 10.1063/1.4804742

Design of a simple low cost tethersonde data acquisition system for meteorological measurements Rev. Sci. Instrum. 76, 084501 (2005); 10.1063/1.1988029 


\title{
A New Challenge for Meteorological Measurements: The "MeteoMet" Project - Metrology for Meteorology
}

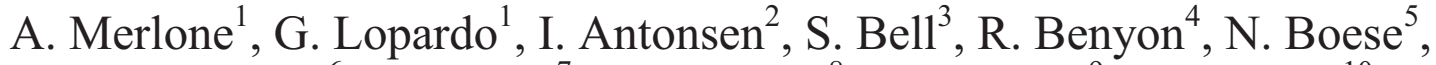 \\ D. del Campo ${ }^{6}$, M. Dobre ${ }^{7}$, J. Drnovšek ${ }^{8}$,A. Elkatmis ${ }^{9}$, E. Georgin ${ }^{10}$, \\ E. Grudniewicz ${ }^{11}$, M. Heinonen ${ }^{12}$, C. Holstein-Rathlou ${ }^{13}$, J. Johansson ${ }^{14}$, \\ P. Klason ${ }^{15}$, R. Knorova ${ }^{16}$, C. Melvad ${ }^{17}$, J. Merrison ${ }^{13}$, K. Migała ${ }^{18}$, \\ M. de Podesta $^{3}$, H. Saathoff ${ }^{19}$, D. Smorgon ${ }^{1}$, F. Sparasci ${ }^{20}$, R. Strnad $^{21}$, \\ A. Szmyrka-Grzebyk ${ }^{22}$, and E. Vuillermoz ${ }^{23}$ \\ ${ }^{1}$ INRiM Istituto Nazionale di Ricerca Metrologica, Strada delle Cacce 73, I-10135 - Turin, Italy \\ ${ }^{2}$ Justervesenet, Fetveien 99, N-2007 Kjelle, Norway \\ ${ }^{3}$ National Physical Laboratory, Hampton Road, Teddington, Middlesex, TW11 OLW, UK \\ ${ }^{4}$ Instituto Nacional de Técnica Aeroespacial, Ctra a Ajalvir, 28850, Torrejon de Ardoz, Spain \\ ${ }^{5}$ Physikalisch-Technische Bundesanstalt, Bundesallee 100, D-38116 Braunschweig, Germany \\ ${ }^{6}$ Centro Español de Metrología, Alfar, 2, ES-28760 Tres Cantos, Madrid, Spain \\ ${ }^{7}$ Federale Overheidsdienst Economie, KMO, Middenstand en Energie - Dienst Wetenschappelijke Metrologie, Bd. \\ Du Roi Albert II, 16, 1000 - Brussels, Belgium \\ ${ }^{8}$ MIRS/UL-FE/LMK Univerza v Ljubjani, Trzaska 25, SI-1000 Ljubljana, Slovenia \\ ${ }^{9}$ TUBITAK Ulusal Metroloji Enstitusu, Gebze Yerleskesi 54, TR-41470 Gebze, Kocaeli, Turkey \\ ${ }^{10}$ Centre Technique des Industries Aérauliques et Thermiques, av. des Arts, Villeurbanne Cedex, France \\ ${ }^{11}$ GUM Glówny Urzad Miar, Plac Trzech Krzyży 3/5 00-507 Warszawa, Poland \\ ${ }^{12}$ MIKES Mittatekniikan Keskus, Tekniikantie 1, P.O. Box 9, FI-02151 Espoo, Finland \\ ${ }^{13}$ Mars Simulation Laboratory, Aarhus University, Ny Munkegade 120, 8000 Aarhus C, Denmark \\ ${ }^{14}$ Chalmers University of Technology, Onsala Space Observatory, SE-43992 Onsala, Sweden \\ ${ }^{15}$ SP Sveriges Tekniska Forskningsinstitut AB, P.O. Box 857, SE-501 15 Borås, Sweden \\ ${ }^{16}$ SMU Slovenský Metrologický Ustav, Karloveská 63, SK-842 55 Bratislava, Slovakia \\ ${ }^{17}$ DTI Teknologisk Institut, Gregersensvej, DK-2630, Taastrup, Denmark \\ ${ }^{18}$ Uniwersytet Wrocławski, ul. Kosiby 6/8, 51-670 Wroclaw, Poland \\ ${ }^{19}$ Karlsruher Institut für Technologie, Kaiserstr. 12, D-76131 Karlsruhe, Germany \\ ${ }^{20}$ Conservatoire national des arts et metiers, rue Saint Martin292,, FR-75141 Paris, France \\ ${ }^{21}$ CMI Cesky Metrologicky Institut Brno, Okružní 31, CZ-638 00 Brno, Czech Republic \\ ${ }^{22}$ Institut Niskich Temperatur i Badan Strukturalnych IM. Wlodzimierza Trzebiatowskiego Polskiej Akadamii Nauk, \\ Okolna str 2, 50-422 Wroclaw, Poland \\ ${ }^{23}$ Comitato EV-K2-CNR, Via San Bernardino 145, 24126, Bergamo, Italy
}

\begin{abstract}
Climate change and its consequences require immediate actions in order to safeguard the environment and economy in Europe and in the rest of world. Aiming to enhance data reliability and reduce uncertainties in climate observations, a joint research project called "MeteoMet - Metrology for Meteorology" started in October 2011 coordinated by the Italian Istituto Nazionale di Ricerca Metrologica (INRiM). The project is focused on the traceability of measurements involved in climate change: surface and upper air measurements of temperature, pressure, humidity, wind speed and direction, solar irradiance and reciprocal influences between measurands. This project will provide the first definition at the European level of validated climate parameters with associated uncertainty budgets and novel criteria for interpretation of historical data series. The big challenge is the propagation of a metrological measurement perspective to meteorological observations. When such an approach will be adopted the requirement of reliable data and robust datasets over wide scales and long terms could be better met.
\end{abstract}

Keywords: air humidity, air pressure, air temperature, air speed and direction, historical temperature data series, meteorological instruments calibration, traceable climate measurements.

Temperature: Its Measurement and Control in Science and Industry, Volume 8

AIP Conf. Proc. 1552, 1030-1035 (2013); doi: 10.1063/1.4821419

(C) 2013 AIP Publishing LLC $978-0-7354-1178-4 / \$ 30.00$ 


\section{INTRODUCTION}

Recent decades have seen notable changes in global climate $[1,2]$. The consequences of these changes have a deep impact on different aspects of social, political and economic life. Reliable assessment of climate change crucially depends on the robustness of climate data and on the uncertainties associated with measurements. The need for focusing the scientific world attention on a different approach to meteorological observations has been clearly stated on various occasions. During an historical meeting held in 2010 [3], the World Meteorological Organization (WMO) and the Bureau International des Poids et Mesures (BIPM) signed the Mutual Recognition Arrangement (MRA) for national measurement standards and for calibration and measurement certificates issued by National Metrological Institutes (NMIs). In this occasion, the two organizations agreed to establish that today many of the principal challenges faced by climate science are indeed measurement challenges and discussed ways for working together. Besides, the CCT (Consultative Committee for Thermometry) Recommendation to CIPM (International Committee for Weights and Measures) [4] encourages a strong cooperation between NMIs and meteorological organizations to work for traceable climate data over long temporal terms and wide spatial scales based on best practice metrology.

In line with these requirements is the European Joint Research Project (JRP) MeteoMet (www.meteomet.org) which will give the opportunity to the metrological scientific community to contribute efficiently in support of environmental policies. Availability of reliable climate data, measurement uncertainty evaluation, robust historical data series, as the project aims, will bring great advantage in term of: better assessment of climate change, development of more accurate predictive models, building of effective adaptation programs.

\section{THE "MeteoMet" PROJECT}

The MeteoMet project started in October 2011 with a three-year lifetime. It is jointly funded by the European Commission and the participating countries within the European Association of National Metrology Institutes (EURAMET) through the European Metrology Research Programme (EMRP) call 2010: Metrology for Environment.

The activities in the project and the specific subject have brought together a wide consortium of partners: 18 NMIs, plus 3 universities as unfunded partners. Not limited to the European area, collaborators joined and follow the JRP advances through signed exchange of letters. Beside Research Institutes, Universities, manufacturers and national Institutions, also intergovernmental Institution and Organizations signed the letters (or started the process leading to the signature); the WMO-CIMO (Commision for Instruments and Methods of Observation), the GRUAN (GCOS Reference Upper-Air Network) and the International Surface Temperature Initiative (ISTI) are a relevant example of such collaboration. Such a wide diverse consortium and stakeholder community represents a key feature of the project, helping to merge different knowledge aimed at improving the actual climate-science evidence basis.

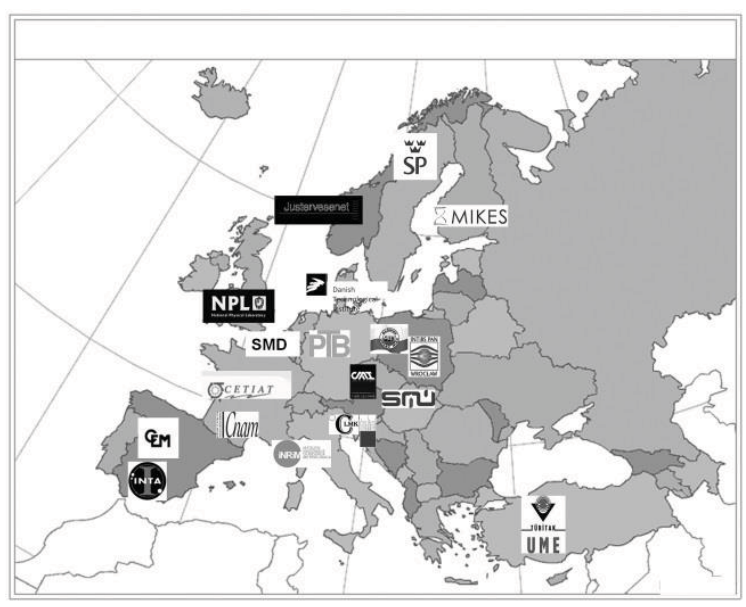

FIGURE 1. NMI partners of MeteoMet

\section{Objectives and Needs for the Project}

The project is focused on the traceability of measurements involved in the climate change evaluation: surface and upper air measurements of temperature $(T)$, pressure $(P)$, humidity $(R H)$, wind speed and direction, solar irradiance and reciprocal influences between measurands. Project main scopes are:

- achievement of robust climate data with measurement uncertainty budget

- accurate interpretation of historical temperature data series.

Moreover, will be developed novel methods and instruments to improve the measurements of $T, P, R H$, and wind speed.

The project aims to strengthen the idea that climate measurements have to be made under standard conditions and in accordance with established practices both for observing methods and for the exposure, selection and use of instruments $[5,6]$. For maintaining a high level of confidence in the climate data, it is necessary to use approved instruments with traceability to International System of Units (SI) 
through the national standards. This is not, however, always defined in Europe [7]. In this scope, NMIs capabilities in defining procedures, calibration standards and traceability chains are vital for achieving significant advancements.

This project, focused on the definition of a European standardization and harmonization, aims to improve communication and co-operation between NMIs and national meteorological institutes or local agencies contributing to climate observations, in order to realize a wide scale monitoring system and to assure the quality of measurements.

Homogeneity and standardization of climate data are desirable and even critical when datasets are used to study questions related to climatic fluctuations and change. However, long-term climatic time series are generally affected by inhomogeneities and statistical data adjustment techniques are usually adopted [8]. The MeteoMet proposal is to shift quality controls before data storage. To have data with associated uncertainty budget allows to classify data respect to their quality and to choose what to record. The availability of traceable measurements, including appropriate uncertainty evaluations, brings to estimate climatic trends in a scientifically more rigorous manner.

Going in the specific topics addressed in the project:

\section{$>$ Upper air measurements}

Numerous humidity sensors have been developed for atmospheric measurements on platforms from ground based to airborne such as planes or balloons, together with basic protocols for their calibration. The very few intercomparisons $[9,10]$ undertaken to validate the performance of field instruments have however revealed: significant discrepancies $(10 \%$ or more) even for the best and well established instruments [9]. Comparing the results and methods shows also a severe lack of consistent calibration protocols and traceability to national humidity standards [9]. In order to provide reliable atmospheric humidity data especially in field conditions, improved calibration instruments and protocols will be developed and established to assure traceability to national standards.

An important influence on the uncertainty of humidity data comes from the saturation vapour pressure equations for water. The uncertainty of the equations will be improved to obtain more consistent humidity data from instruments based on different principles of operation. The results will also be benefitted in relevant humidity calibrations against primary humidity standards.

The development of radiosondes to fulfill the data accuracy requirements for studying earth's climate variability has been identified as a focus working area by GRUAN [6, 11]. A key factor in achieving the required reliability will be the traceability of radiosonde sensor measurements through appropriate calibration. This JRP will develop a novel calibration system for improving the efficiency of humidity and temperature calibrations of radiosondes.

\section{$>$ Ground based measurements}

At present, many weather stations are not equipped with approved standard instruments, and robust traceability to national standards is missing. Furthermore, a general lack of analysis of the mutual influences of the monitored parameters currently affects the performances of those devices. These influences will be studied using an existing $P-T-R H$ calibration system [12] and new facilities to be developed in this project for the traceable calibration of weather stations in situ and in laboratory.

Sensitivity to exposure to wind and solar irradiance, sampling rates and operation environments are fundamental parameters which will be considered and standardized. In addition, accurate methods for insitu calibrations of weather stations, including those operating in extreme weather conditions, will be defined.

Assessment of the uncertainties of historical temperature measurement data

An interesting and controversial theme is the ability to compare historical and modern data series. The surrounding environment conditions, uncertainty budgets and traceability to standards and temperature scales, make it difficult to assess the reliability of the data and to compare the data of the different periods. The project intends to establish criteria for the validation of different spatial and temporal data, investigating the sources of uncertainty affecting the historical temperature data series.

\section{Project Issues}

The JRP structure reflects two main aspects of metrology: scientific innovation and practical traceability for end users. It includes development and testing of novel instruments as well as improved calibration procedures and facilities for ground based observations, in-situ practical calibrations and best practice dissemination. The work addresses the following topics:

\section{$>$ Upper air measurements}

During the project a new generation of humidity sensors, both laser and microwave based, will be developed. 
Tuneable Diode Laser Absorption Spectroscopy (TDLAS) hygrometers offer the opportunity for rapid, highly selective analysis of water in air. But their major challenge as traceable absolute hygrometers has not yet been developed. Furthermore, full uncertainty budgets for calibrated field laser hygrometers are missing. The instruments to be developed during the project will be validated against the German national humidity standard and their uncertainties analyzed.

Open path measurements covering the troposphere and the stratosphere are very demanding due to the large pressure, temperature and humidity range which occur in a vertical atmospheric profile. In this project traceable spectral data (line strength and width) of the water molecule will be determined for the first time, including their pressure / temperature and matrix dependence, with the aim of overcoming the discrepancies evident in the literature.

Microwave quasi-spherical resonant cavities hygrometers would provide a unique instrument able to perform measurements from the lower troposphere up to the stratosphere with very high sensitivity to the water vapor concentration and high measurement accuracy. A compact, robust and high-sensitive microwave quasi-spherical cavity suitable for installation in airborne devices will be developed to measure humidity in the upper atmosphere, where high accuracy is needed at water vapour concentration levels.

Moreover, in order to improve comparability between existing sensor technologies this project will carry out:

- development of a new mobile humidity generator to enable onsite calibration of field hygrometers

- development of a new "fast humidity calibration system" for establishing traceability to radiosonde-based measurements

- the $2^{\text {nd }}$ international intercomparison campaign (Aquavit 2) [9] of airborne field humidity sensors of different types using the AIDA (Aerosol Interaction and Dynamics in the Atmosphere) chamber of the Karlsruhe Institute of Technology (KIT) in Germany. This activity will be closely coordinated with the European Cooperation in Science and Technology (COST) Action ES0604: Atmospheric Water Vapour in the Climate System (WaVaCS), the SPARC (Stratospheric Processes And their Role in Climate) water vapour initiative (a project of the World Climate Research Program), and GRUAN.

In order to provide new instruments and methods devoted to the measurement of $T, P$ and $R H$ in the lower and upper atmosphere, this JRP includes the development of innovative multisensors for free-space non-contact atmospheric measurements and novel methods for GPS (Global Positioning System) and Galileo-based measurements.

Multi-approach sensing will enable rapid simultaneous measurements of the same air mass, whilst reducing the influence of the sensors themselves on the measurements. The newly developed instrument systems will be used on combined balloon-borne platforms.

Water vapour in the atmosphere is difficult and costly to measure with high temporal and spatial resolution, due to its large variability. Signal from GNSS (Global Navigation Satellite System) represents an efficient solution for measuring water vapor concentration and retrieve vertical profiles of $T$ and $P$.

\section{Ground based measurements}

Three basic aspects related to weather station based measurements will be covered:

- Proposal for novel calibration methods and protocols.

In this context the first metrological intercomparison and testing of weather stations will be organized.

The validation of data-logging software used to calculate the indirect measured value from the direct measurement will be performed. Its validation aims to avoid measurement processing errors.

- Evaluation of the effect of solar radiance and wind speed on meteorological parameters measurements.

Based on this information a "standard" radiation shield will be developed in order to improve the accuracy and uncertainty of air temperature measurements. The proposal of procedures for harmonizing measurements with different solar radiation shields will be included. A theoretical model for studying the influence of solar radiation on weather measurements and for uncertainty budget evaluation taking into account aging effects will be developed.

One of the most measured parameters in weather stations is the wind. However, the measurements performed are difficult to use, primarily due to the influence of the environment. Methods for on-site field calibrations will be developed addressing the influence from the geographical layout. Further the effect of foreign substances (e.g. rain and icing) on ultrasonic anemometers will be optimized using a validated mathematical model. 
- Construction of facilities for laboratory and in situ calibration of weather stations also working under extreme environmental conditions.

This project aims to develop dedicated facilities for the combined and simultaneous calibration, in situ and in laboratory, of $T, P$ and $R H$ sensors in weather stations. A laboratory chamber will contain also a wind generator and will be designed to include a solar radiation generator.

The facility developed for in situ calibration will have reduced dimensions in order to be adaptable for the calibration of weather stations and instruments used for the monitoring of environmental parameters also in high altitudes. The new facility is expected to bring benefits to usual weather measurements and high altitude monitoring programs which are important indicators of climate change. The innovative transportable calibration chamber will be used for weather stations operating in the EV-CNRK2 Pyramid Laboratory/Observatory at the base of Mount Everest in Nepal and at research stations in the Karkonosze Mountains and in the Svalbard Island.

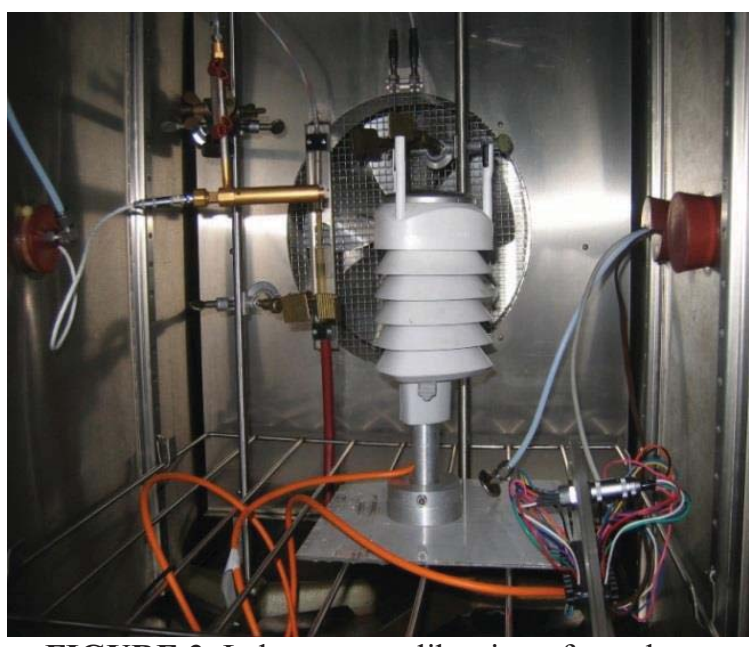

FIGURE 2. Laboratory calibration of weather station at INRiM

Assessment of the uncertainties of historical temperature measurement data

The principal aim is to reduce the variability of historical temperature data series and to develop novel software for the harmonisation of data under a metrological approach. Evaluation of temperature trends will be carried out analyzing Type A and Type B uncertainties.

\section{CONCLUSIONS}

This JRP will provide the first definition of metrologically-validated parameters for use as climate indicators. This objective will be obtained through the definition of new measurement protocols and calibration procedures in accordance with WMO guidance, measurement uncertainty evaluation methods, and more robust historical temperature data series.

During the project, the definition of a set of calibrated weather stations and radiosondes traceable to national standards will be supported. The development of novel instruments will be a scientifically and technically significant result of the project as well as a stimulus for innovation.

Attention to these objectives is clearly stated by the participation of instrument manufacturers as collaborating stakeholders. The new instruments will be provided to the end users with a complete metrological characterization, which includes the identification of the parameters to be taken into account in the determination of the uncertainty budgets associated to the measurements and adequate calibration procedures necessary to ensure traceability to the standards at the required level of uncertainty.

Then, the project aims to improve availability of traceable data and promote their use, improve metrological best practice dissemination and communication and co-operation within scientific community.

Building a European centre of excellence for climate monitoring is the big challenge of the project. The creation of a network of meteorological institutes linked to NMIs will be supported. This network through the outputs of the JRP will be able to provide more accurate and reliable observations of earth's climate. The large number of partners and collaborators involved in the project will help to know diverse needs in order to discuss and propose common procedures fitting different requirements. NMIs operating at a regional level in cooperation with meteorological institutes will be able to disseminate more efficiently and directly the JRP results and metrological best practices with the awareness that reliable climate data is a geographically equally relevant matter.

The cooperation between Metrology and Meteorology supported in this project will improve both scientific communities. This aspect, meeting the requirements stated in CCT Recommendation [4] and the conclusion of BIPM-WMO Workshop [3], will open a new era for meteorological measurements. 


\section{ACKNOWLEDGEMENTS}

The research leading to these results has received funding from the EMRP participating countries within EURAMET and the European Union

\section{REFERENCES}

1. Fourth Assessment Report of the Intergovernmental Panel on Climate Change "Climate Change 2007 - The Physical Science Basis" (2007).

2. Blunden, J., Arndt D. S., and Baringer M. O., "State of the Climate in 2010". Bull. Amer. Meteor. Soc., 92 (6), S1-S266 (2011).

3. WMO-BIPM workshop on: "Measurements Challenges for Global Observation Systems for Climate Change Monitoring" 30 Mar. 2010 Geneva, Switzerland.

4. CCT Recommendation to CIPM T3 (2010) on climate and meteorological observations measurements - Doc. $\mathrm{CCT} / 10-09$

5. Guide to meteorological instruments and methods of observation (WMO-No.8) (2008)

6. Reference Upper-Air Observations for Climate: Rationale, Progress, and Plans

7. Seidel D. J., Berger F. H., Immler F., Sommer M., Vömel H., Diamond H. J., Dykema J., Goodrich D., Murray W., Peterson T., Sisterson D., Thorne P., Wang J., Bulletin of the American Meteorological Society 90, 361-369 (2009).

8. Merlone, A., Meda, A., Pennecchi, F. R., Sardi, M., Measurement 42, 10 1482-6 (2009)

9. Menne, M. J., Williams, C. N. Jr., Journal of Climate 22, 1700-1717 (2009).

10. Fahey, D., Gao, R., Möhler O., "The AquaVIT white paper", 2009. https://aquavit.icg.kfa-juelich.de/AquaVit/

11. Nash J., Oakley T., Vömel H., LI Wei, "8th WMO Intercomparison of High Quality Radiosonde Systems, Yangjiang, China, 12 - 31 July 2010" (WMO-CIMO Report No. 107)

12. Report of the GRUAN Implementation Meeting, GCOS121 (WMO/TD No. 1435), WMO 2008

13. Saxholm, S., Heinonen, M., Measurement 43, 1583-8 (2010). 